\title{
A phase II and pharmacodynamic study of sunitinib in relapsed/refractory oesophageal and gastro-oesophageal cancers
}

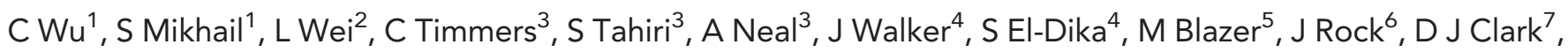
$X$ Yang $^{7}$, J L Chen ${ }^{8}$, J Liu ${ }^{9}, M V$ Knopp ${ }^{7}$ and T Bekaii-Saab*,1

${ }^{1}$ Division of Medical Oncology, Department of Internal Medicine, The Ohio State University Comprehensive Cancer Center, Arthur G James Cancer Hospital and Richard J Solove Research Institute, Columbus, OH 43210, USA; ${ }^{2}$ Center for Biostatistics, The Ohio State University Comprehensive Cancer Center, Columbus, OH 43210, USA; ${ }^{3}$ The Ohio State University Comprehensive Cancer Center, Arthur G James Cancer Hospital and Richard J Solove Research Institute, Columbus, OH 43210, USA; ${ }^{4}$ Division of Gastroenterology, Hepatology and Nutrition, Department of Internal Medicine, The Ohio State University Wexner Medical Center, Columbus, OH 43210, USA; ${ }^{5}$ Department of Pharmacy, The Ohio State University Wexner Medical Center, Columbus, $\mathrm{OH}$ 43210, USA; ${ }^{6}$ Anatomic Pathology Laboratory, Mercy Anderson Hospital, Cincinnati, OH 43210, USA; ' Department of Radiology, The Ohio State University Wexner Medical Center, Columbus, OH 43210, USA; ${ }^{8}$ Division of Medical Oncology, Departments of Biomedical Informatics and Internal Medicine, The Ohio State University Comprehensive Cancer Center, Arthur G James Cancer Hospital and Richard J Solove Research Institute, Columbus, OH 43210, USA and ${ }^{9}$ Department of Pathology, Beaumont Medical Center, Grosse Pointe, MI, USA

Background: Blockade of the vascular endothelial growth factor (VEGF) pathway shows evidence of activity in gastro-oesophageal (GE) and oesophageal cancer. We investigated the efficacy of sunitinib, a multikinase VEGF inhibitor, in patients with relapsed/ refractory GE/oesophageal cancer.

Methods: This was a single-stage Fleming phase II study. The primary end point was progression-free survival (PFS) at 24 weeks. If five or more patients out of a total of 25 were free of progressive disease at 24 weeks, sunitinib would be recommended for further study. Patients received sunitinib $37.5 \mathrm{mg}$ orally daily and imaged every 6 weeks. Exploratory correlative analysis included serum growth factors, tumour gene expression and dynamic contrast-enhanced magnetic resonance imaging (DCE-MRI).

Results: Twenty-five evaluable patients participated in the study. Progression-free survival at 24 weeks was $8 \%$ ( $n=2$ patients; confidence interval (Cl): 95\% 1.4-22.5\%), and the duration of best response for the patients was 23 and 72 weeks. Ten patients (42\%) had stable disease (SD) for $>10$ weeks. Overall response rate is $13 \%$. Median PFS is 7 weeks (95\% Cl: $5.6-11.4$ weeks) and the median overall survival is 17 weeks (95\% Cl: 8.9-25.3 weeks). Most common grade 3/4 toxicities included fatigue (24\%), anaemia (20\%) thrombocytopenia (16\%), and leucopenia (16\%). No patients discontinued therapy due to toxicity. Serum VEGF-A and -C levels, tumour complement factor B (CFB) gene expression, and DCE-MRI correlated with clinical benefit, defined as SD or better as best response.

Conclusion: Sunitinib is well tolerated but only a select subgroup of patients benefited. Serum VEGF-A and -C may be early predictors of benefit. On this study, patients with clinical benefit from sunitinib had higher tumour CFB expression, and thus has identified CFB as a potential predictor for efficacy of anti-angiogenic therapy. These findings need validation from future prospective trials.

*Correspondence: Dr T Bekaii-Saab; E-mail: Tanios.Bekaii-Saab@osumc.edu

Contents of this manuscript have been presented at the 2014 Gastrointestinal Cancers Symposium in a poster presentation.

Received 17 February 2015; revised 18 April 2015; accepted 7 May 2015; published online 7 July 2015

(c) 2015 Cancer Research UK. All rights reserved 0007-0920/15 
Cancers involving the oesophagus and the gastro-oesophageal (GE) junction are the eighth most common cancer in the world (Ferlay et al, 2012). The overall survival (OS) rates for patients with GE cancers remain dismal with survival measured in months.

Blockade of the vascular endothelial growth factor (VEGF) pathway in GE cancer remains a valid therapeutic strategy. There are three known VEGF receptors (VEGFRs): VEGFR-1, -2, and -3 with VEGFR-2 being the main effector of angiogenesis. Patients with oesophageal cancer were found to have significantly higher levels of serum VEGF when compared to controls (De Vita, 2010). Vascular endothelial growth factor receptor levels correlate with a more aggressive behaviour in GE cancers, likely through promoting paracrine and autocrine pathways involved in tumour metastasis (Zhang et al, 2002). Blocking angiogenesis is therefore an attractive strategy to inhibit tumour growth, invasion, and metastasis in GE cancers. Recently, positive results from two randomised clinical trials with ramucirumab (Cyramza-Lilly, Indianapolis, IN, USA), a monoclonal antibody to VEFGR-2, suggest that inhibition of the VEGF pathway remains a valid treatment approach (Fuchs et al, 2014 and Wilke et al, 2014).

Sunitinib (Sutent-Pfizer, New York, NY, USA) is a multikinase inhibitor of mainly VEGFR-1, -2, and -3, and PDGFR with documented activity in a variety of tumours (Gan et al, 2009). In preclinical studies, VEGF-A stimulated proliferation of VEGFR-2 expressing gastric cancer cell lines and sunitinib effectively inhibits cell growth in a dose-dependent manner Lyros et al, 2010). Xenograft tumours in mice treated with sunitinib were found to have decrease in tumour volume and Ki67-staining cells (Zhang et al, 2014). Thus, we hypothesised that sunitinib would be active in patients with GE cancers. For our exploratory correlative studies, we analysed serial serum levels of VEGF-A-D, VEGFR-2 and -3 , and placenta growth factor (PlGF) to determine whether their levels correlated with prognosis or were predictive of therapy benefit. Tumour gene expression profiling was performed to identify differential gene expressions in patients who did $v s$ who did not benefit from sunitinib. In addition, dynamic contrastenhanced magnetic resonance imaging (DCE-MRI) was performed to assess the acute effects of sunitinib on tumour vascularity and viability. Herein, we present the results of a phase II study of sunitinib in patients with relapsed/refractory with oesophageal and GE cancers.

\section{PATIENTS AND METHODS}

Eligibility. Patients were required to have unresectable pathologically confirmed oesophageal or GE cancer, measurable disease per RECIST 1.0, no $>2$ lines of prior palliative therapy, no prior anti-VEGF therapy, ECOG performance status of $\leqslant 1$, no cardiac dysrhythmias, normal or well-controlled blood pressure, normal or well-controlled thyroid function, and no warfarin.

Study design. This phase II study protocol was approved by the institutional review board at The Ohio State University, and the registered trial number is NCT00702884. Informed consent was obtained from each patient. The primary end point was to assess PFS rate at 24 weeks. The secondary end points included overall response rate, median OS, and toxicities. Correlative end points assessed tumour transcriptome expression, changes in vascularity via DCE-MRI, and serum angiogenesis markers.

Sunitinib administration and dose modification. Patients received sunitinib $37.5 \mathrm{mg}$ by mouth daily, with two planned dose-reduction levels (25 and $12.5 \mathrm{mg}$ ).

Assessment of response and toxicity. Tumours measurements were assessed by CT scans every 6 weeks according to RECIST criteria (Therasse et al, 2000). Toxicities were graded according to the NCI Common Toxicity Criteria version 3.0 (http:// ctep.cancer.gov/protocolDevelopment/electronic_applications/ docs/ctcaev3.pdf).

Serum molecular correlates. Blood samples were collected before starting sunitinib at weeks 2 and 6 of treatment, and after disease progression. Commercial ELISA kits were utilised for serum measurement of VEGF (A, C, and D), VEGFR (2 and 3), and PlGF.

Tumour gene expression analysis. Total RNA was extracted from tumour samples using the Norgen Biotek FFPE RNA purification kit. Whole-transcriptome profiling was performed using the SensationPlus FFPE kit and Affymetrix HTA 2.0 array. Exon-level normalisation was performed followed by gene-level summarisation.

Advanced imaging. Longitudinal DCE-MRI studies were performed at weeks $0,2,6$, and 12 to track changes in tumour volume, perfusion, and vascular permeability. Magnetic resonance imaging studies were performed with a 3-Tesla scanner (Achieva; Philips Healthcare, Cleveland, OH, USA) using a 16- or 32-channel phased-array coil and multi-transmit. The DCE-MRI data acquired in the axial and coronal planes were analysed with specialised software written in IDL (Exelis, Boulder, Co., Boulder, CO, USA). Pharmacokinetic analysis was performed on the tracer kinetic curves derived from manually traced, motion-compensated regions of interest using the adjusted Brix's model (Yang et al, 2008).

Statistical methods. This study was a single-stage Fleming phase II study with 25 patients. If $5 \geqslant$ patients were progression free at 24 weeks, sunitinib would be recommended for further investigation $\left(a=0.10, b=0.10 ; P_{0}=0.10, P_{1}=0.30\right)$. The historical control is from a phase II study of gefitinib in second-line therapy of advanced oesophageal cancer, and results showed that of the 26 patients evaluated, treatment was $>8$ weeks in 15 out of 26 patients (58\%; Van Groeningen et al 2004). Thus, time to progression would be 10 weeks or $17 \%$ progression free at 24 weeks. Survival curves were estimated using the Kaplan-Meier method, and 95\% confidence intervals (CI) for the medians are provided. Log-rank test was used to compare PFS between the patients with clinical benefit (non-progressors) vs disease progression. Secondary end points were evaluated using descriptive statistics. Linear mixed effect model was used to examine the correlation between clinical benefit and serum levels for VEGF (A, C, and D), PIGF, and VEGFR (2 and 3) through time (baseline, 2 weeks, 6 weeks, and 12 weeks). Dynamic contrast-enhanced magnetic resonance imaging pharmacokinetic parameters and RECIST size measurements were studied through time using linear mixed effect models. For tumour gene expression analysis, paired $t$-test analysis was performed based on desired response parameters to generate a nominal $P$-value.

\section{RESULTS}

Patient characteristics. Twenty-five evaluable patients were enrolled and evaluable for toxicities (Table 1). Twenty-four patients were evaluable for response. All patients were evaluable for OS and PFS.

Treatment toxicity. Most common grade 3 toxicities included fatigue (24\%), anaemia (20\%), and leucopenia (16\%) (Table 2). There were no grade 4 non-haematological toxicities. Dose reductions were necessary for three patients due to grade 2 pain, grade 3 thrombocytopenia, and grade 3 anaemia. No patients came off of study due to toxicities.

Treatment efficacy. Progression-free survival at 24 weeks was $8 \%$ (two patients; 95\% CI: 1.4-22.5\%) (Table 3 and Figure 1). Three patients had partial response (PR), and their duration of best 


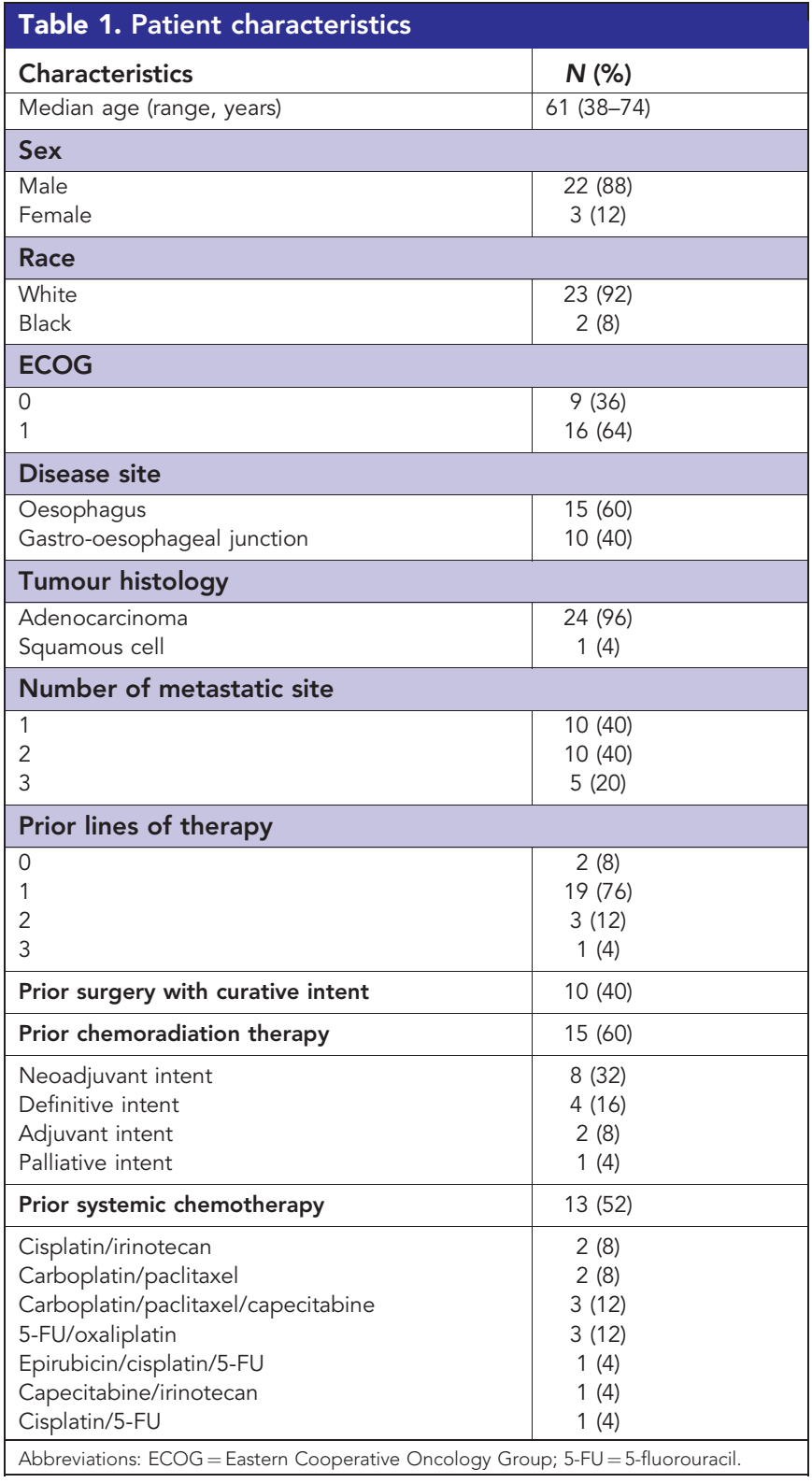

response was 16, 23, and 72 weeks. The patient who had PR for 16 weeks initially presented with a T1 oesophageal adenocarcinoma that was surgically resected, but then developed local recurrence with metastatic lymph node and omental disease 6 months after his surgery. He received sunitinib as first-line therapy. The second patient with PR for 23 weeks was diagnosed with metastatic squamous cell oesophageal cancer with widely metastatic nodal disease in the chest and abdomen, and sunitinib was also her firstline therapy. The final patient presented with metastatic GE adenocarcinoma with liver involvement. He was initially treated on a clinical trial with FOLFOX and vismodegib, and then treated with sunitinib as second-line therapy. Nine patients had stable disease (SD), including seven with SD lasting $>10$ weeks. Most patients with SD had relative shrinkage or no growth of their target lesions (Figure 1).

\section{Correlative studies}

Serum marker analysis

Correlation between baseline VEGF-A and -C levels and treatment response: Serum levels for VEGF (A, C, and D), PIGF, and VEGFR (2 and 3) were obtained in 24 patients (Figure 2 and
Table 2. Related toxicities

\begin{tabular}{|c|c|c|c|}
\hline & $\begin{array}{c}\text { Grade } 1 / 2 \\
N(\%)\end{array}$ & $\begin{array}{c}\text { Grade } 3 \\
N(\%)\end{array}$ & $\begin{array}{c}\text { Grade } 4 \\
N(\%)\end{array}$ \\
\hline \multicolumn{4}{|l|}{ Haematological } \\
\hline Leukopenia & $8(32)$ & $3(12)$ & $1(4)$ \\
\hline Lymphopenia & $4(16)$ & $3(12)$ & 0 \\
\hline Neutropenia & $6(24)$ & $2(8)$ & $1(4)$ \\
\hline Anaemia & $12(48)$ & $3(12)$ & $2(8)$ \\
\hline Thrombocytopenia & $3(12)$ & $3(12)$ & $1(4)$ \\
\hline \multicolumn{4}{|c|}{ Non-haematological } \\
\hline Anorexia & $12(48)$ & 0 & 0 \\
\hline Diarrhoea & $11(44)$ & 0 & 0 \\
\hline Fatigue & $13(52)$ & $6(24)$ & 0 \\
\hline Mucositis & $7(28)$ & 0 & 0 \\
\hline Hypertension & $2(8)$ & $1(4)$ & 0 \\
\hline Haemorrhage & $6(24)$ & $1(4)$ & 0 \\
\hline Hyperglycaemia & $6(24)$ & $2(8)$ & 0 \\
\hline Abdominal pain & $10(40)$ & $2(8)$ & 0 \\
\hline Nausea & $13(52)$ & $1(4)$ & 0 \\
\hline Vomiting & $9(36)$ & 0 & 0 \\
\hline
\end{tabular}

\section{Table 3. Efficacy results}

\begin{tabular}{|l|c|c|}
\hline End point & $\mathbf{N}(\%)$ & $95 \% \mathrm{Cl}$ \\
\hline PFS at 24 weeks & $2(8)$ & $1.4-22.5 \%$ \\
\hline Median PFS (weeks) & 6.9 & $5.6-11.4$ \\
\hline Median OS (weeks) & 16.6 & $8.9-25.3$ \\
\hline ORR & $3(12.5)$ & \\
\hline PR & $9(37.5)$ & \\
SD & $7(29)$ & \\
SD > 10 weeks & $10(42)$ & \\
DCR (PR + SD > 10 weeks) & $12(50)$ & \\
PD & \multicolumn{2}{|l|}{} \\
\hline Abbreviations: Cl=confidence interval; ORR = overall response rate; OS = overall survival; \\
PFS = progression-free survival; PR=partial response; SD = stable disease. \\
\hline
\end{tabular}

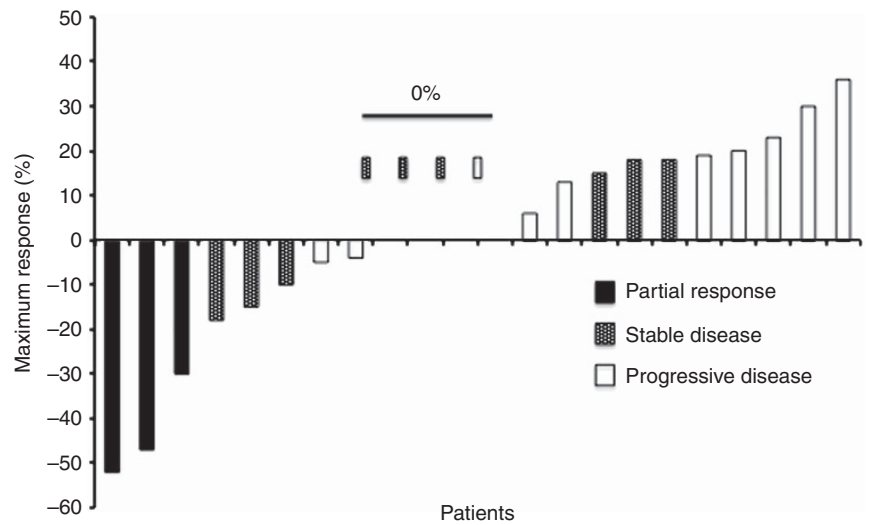

Figure 1. Waterfall plot of patients' best response.

Supplementary Table 1). Patients who had clinical benefit had higher VEGF-C baseline values $(P=0.04)$. In addition, VEGF-C levels were decreased at 2 weeks $(P=0.03)$ and 6 weeks $(P=0.01)$. Conversely, patients with $\mathrm{PD}$ were observed to have an increase in serum VEGF-A levels at weeks 2 and $6(P=0.02$ for both time points). Patients with PD had an increase in serum levels of PlGF from baseline to 2 weeks $(P=0.0003)$ and a trend for continued increase at week 6 . VEGFR-2 was decreased at weeks 2 and 6 in both groups. 

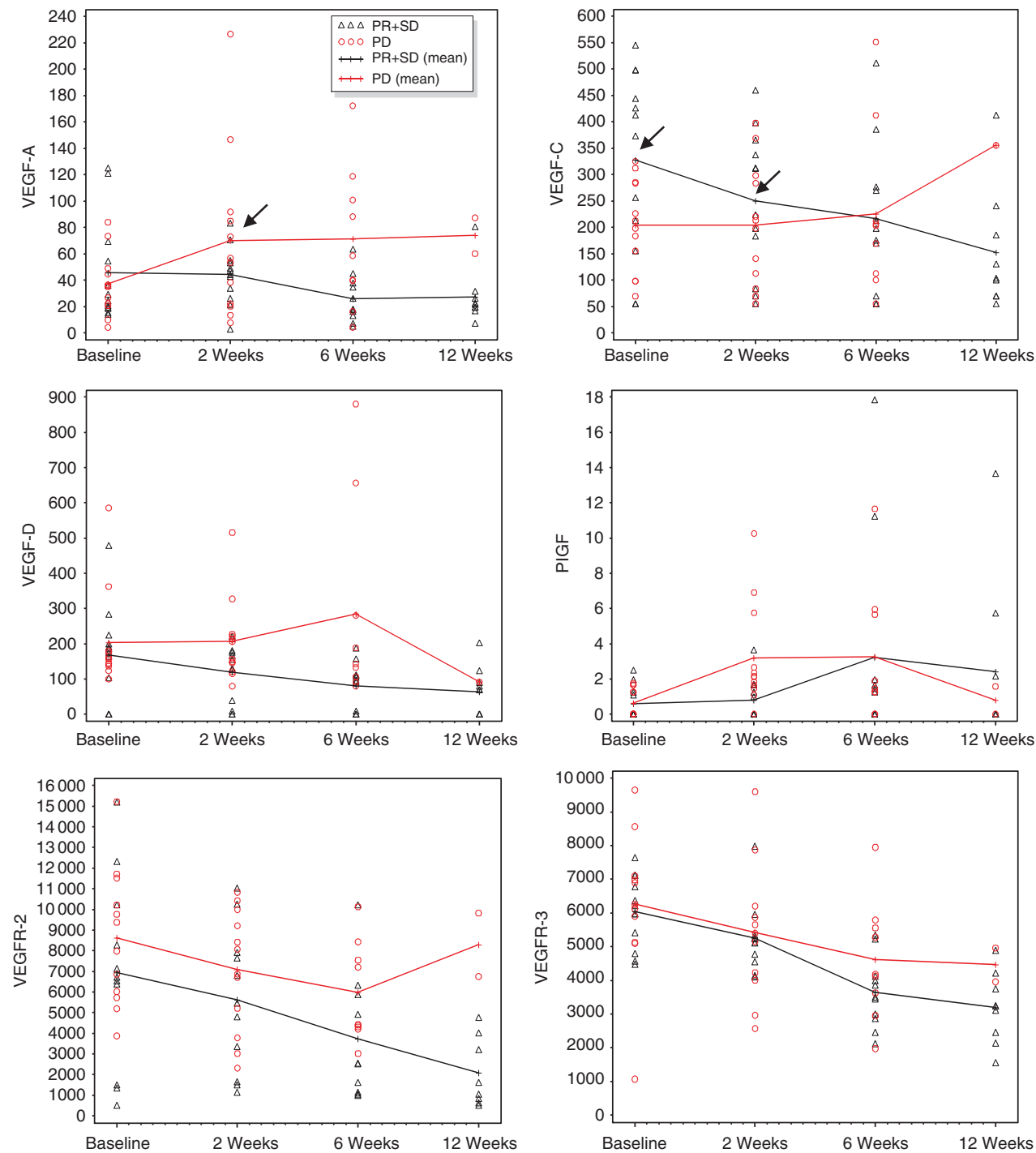

Figure 2. Serum growth factor levels. Patients who had PD are in red and patients who had clinical benefit (SD or PR) are in black. Patients with PD were found to have significantly higher VEGF-A levels at week 2, as compared with baseline. Patients who did derive clinical benefit from sunitinib were found to have statistically significant higher VEGF-C levels at baseline, and decrease at 2 weeks.

\section{Gene transcriptome profile}

Correlation between gene expression profiling and clinical benefit: Sixteen formalin-fixed, paraffin-embedded biopsy of tumours from 15 patients were obtained from patients before starting sunitinib therapy were evaluable for gene expression profiling (Figure 3 and Supplementary Table 2). There were 44699 gene transcript clusters evaluated between the six patients who derived clinical benefit and nine who had PD. There were 47 differentially expressed transcript clusters in coding regions (nominal $P \leqslant 0.05$ ). A nominal $P$-value was chosen given that this was exploratory in nature. There were 12 transcript clusters that mapped to known genes. Seven of these 12 transcript clusters corresponded to complement factor B (CFB) with a median fold-change of 1.71. A significant trend for increased CFB expression was found in the six patients who had clinical benefit with improved PFS but not OS (Supplementary Table 2).

Advanced Imaging results. Dynamic contrast-enhanced magnetic resonance imaging pharmacokinetic parameters and RECIST size measurements were analysed in 16 patients (Supplementary Figure 1). The difference in baseline mean tumour size and the MRI parameters were not significantly different between the two patient groups: (patients with PD patients with clinical benefit) tumour size $6.77 \mathrm{~cm} \quad(P=0.79), \quad$ Amp $0.56 \quad(P=0.22)$, $K_{\mathrm{ep}} 1.53 \mathrm{~min}^{-1}(P=0.18), K_{\mathrm{pe}}-0.04 \mathrm{~min}^{-1}(P=0.97)$, and $K_{\mathrm{el}}$ $0.02 \mathrm{~min}^{-1}(P=0.50)$. Patients with clinical benefit had stable mean tumour size over the initial 12 weeks, suggesting a cytostatic response to sunitinib. This is supported by the flat trend observed in the Amp values that indicate sunitinib did not induce any substantial change in tumour perfusion. The plasma-toextracellular space transfer rate $K_{\text {pe }}$ shows a decrease at week 2 in patients with clinical benefit, although this is not statistically significant $(P=0.14)$, likely a result of sunitinib-induced normalisation of tumour microvasculature.

\section{DISCUSSION}

Patients with oesophageal and GE cancers have poor outcomes. Despite some early disappointments with targeting the VEGF pathway with bevacizumab (Avastin-Roche/Genentech, San Francisco, CA, USA), a monoclonal antibody blocking VEGF-A ligand, recent trials with ramicurumab in patients with refractory gastric cancer showed an improvement in outcome (Shah et al, 2006, Fuchs et al, 2014, Wilke et al, 2014, and Ohtsu et al, 2011). Another recent study suggested a significant benefit in 


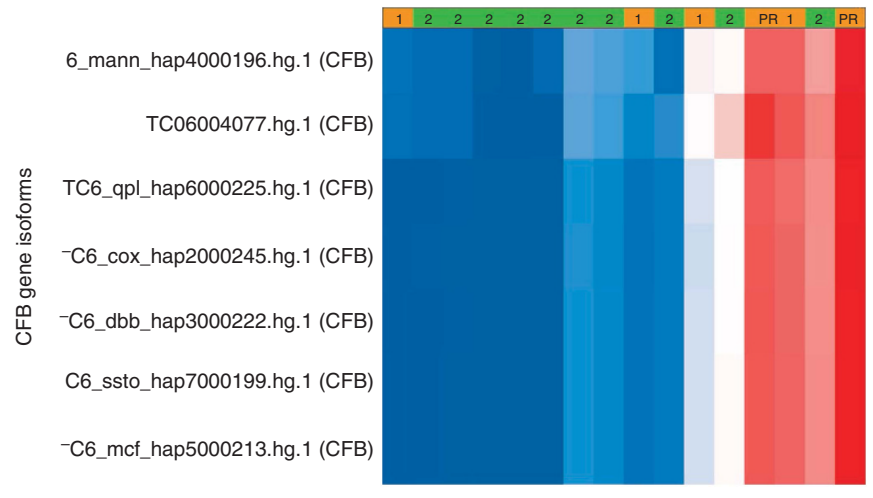

Figure 3. Heat map of CFB gene isoform expression levels. Patients who did not have disease progression (SD + PR) are depicted in orange, whereas those with PD are in green. Expression levels red $=$ high, blue $=$ low. Collectively, there was a statistical trend towards higher expression of CFB in those patients who benefited from VEGFR inhibition.

gastric cancer with apatinib, a multikinase inhibitor similar to sunitinib vs best supportive care (Qin, 2014).

There have been no reported prior studies of single-agent sunitinib in oesophageal and GE cancers, although sunitinib has been given in combination with chemotherapy with no improved PFS. A phase II study from the Hoosier Oncology Group treated 28 patients with advanced oesophageal cancer with the combination of sunitinib ( $37.5 \mathrm{mg}$ orally daily) and paclitaxel $\left(90 \mathrm{mg} \mathrm{m}^{-2}\right.$ intravenously on days 1,8 , and 15 of a 28-day cycle). Results showed that PFS at 24 weeks was $25 \%$ (90\% CI: $12-42 \%$ ), which was not improved from historical control (Schmitt et al, 2012). In addition, a randomised placebo-controlled phase II study of FOLFIRI with or without sunitinib in 91 patients with advanced GE cancers showed that median PFS was similar in the sunitinib vs placebo arm (3.6 vs 3.3 months, respectively, HR 1.11, 95\% CI: 0.70-1.74, $P=0.66$; Moehler et al, 2013).

Sunitinib was well tolerated at a daily oral dose of $37.5 \mathrm{mg}$, no one came off of trial due to toxicities. Although our study did not achieve its primary end point, the presence of objective durable responses in three patients is encouraging. The overall outcome in our study is comparable to other studies in gastric and GE cancer (Fuchs et al, 2014 and Wilke et al, 2014)

Our correlative studies were performed to identify potential prognostic or predictive biomarkers. The DCE-MRI data support that patients who derived clinical benefit from sunitinib had a decrease in tissue perfusion. VEGR-2 and -3 serum levels were decreased from baseline in all patients at weeks 2 and 6 of treatment. This likely reflects drug inhibition and may not necessarily correspond with disease response (Grivas et al, 2014).

Placenta growth factor, a ligand for VEGFR-1, had a significant increase at week 2 in patients who had PD. However, there was a trend for increase in PlGF over time in all patients, which could be due to inhibition of VEGFR. In the AVAGAST trial, high baseline plasma levels of VEGF-A were suggested to be predictive of sensitivity to bevacizumab in Western patients (Van Cutsem et al, 2012). In our study, we noticed that the trend of stable VEGF-A levels rather than the baseline level was indicative of treatment response. Patients with PD had an increase in their VEGF-A levels at weeks 2 and 6 of treatment. In contrast, patients who had clinical benefit did not have an increase in subsequent VEGF-A levels. Interestingly, patients who had clinical benefit had higher levels of serum VEGF-C at baseline and a significant decrease in serum levels at weeks 2 and 6 of therapy. A similar finding with sunitinib was documented in renal cell carcinoma but only with baseline
VEGF-C levels (Rini et al, 2008). As such, our findings with serum VEGF-A and -C levels may be early predictor of clinical benefit of VEGF-directed therapy.

Tumour transcriptome analysis revealed that differential CFB expression was observed in patients who derived clinical benefit from sunitinib vs those with PD. CFB circulates in the blood and is involved in the normal activation of the alternative complement pathway. It is cleaved by complement D to yield two subunits, $\mathrm{Ba}$ and $\mathrm{Bb}$, which are involved in the proliferation of $\mathrm{B}$ lymphocytes. CFB has been found to be important for driving inflammation and angiogenesis via secretion of VEGF. CFB has been studied in the pathogenesis of age-related macular degeneration (AMD), a disease that is typically treated with anti-angiogenic agents such as aflibercept and bevacizumab (Selid et al, 2014). In mouse models for AMD, laser treatment to the Bruch's membrane, the innermost layer of the choroid in the eye, showed increase production of angiogenic factors. However, mice treated with siRNA to CFB had low levels of VEGF and TGF-B2 after laser treatment, demonstrating the significance of CFB in the angiogenesis pathway (Bora et al, 2006). Based on our results, we hypothesise that sunitinib may block CFB or inflammationdependent VEGF release in GE tumours. Elevated CFB transcript levels may predict for response to sunitinib and other VEGFdirected therapy.

In conclusion, sunitinib is well tolerated but seems to benefit only a subgroup of patients. It is important to identify a biomarker that will predict response to therapy, and our correlative studies have found possible candidates although further validation is needed. Changes in baseline serum levels of VEGF-A and -C may serve as early predictors of clinical benefit with significant potential implications for the design of future trials. More interestingly, our study includes the first report of differential CFB expression as a potential predictor for efficacy of anti-angiogenic therapy in a solid tumour, and may warrant further investigation in future trials with anti-VEGF drugs.

\section{ACKNOWLEDGEMENTS}

This study has been funded by Pfizer and The Ohio State University Comprehensive Cancer Center GI Oncology Research Fund. CW is funded on K12 Faculty Training Grant - CA133250.

\section{CONFLICT OF INTEREST}

TB-S is a paid consultant of Lilly, Pfizer, and Genentech. The remaining the authors declare no conflict of interest.

\section{REFERENCES}

Bora NS, Kaliappan S, Jha P, Xu Q, Sohn JH, Dhaulakhandi DB, Kaplan HJ, Bora PS (2006) Complement activation via alternative pathway is critical in the development of laser-induced choroidal neovascularization: role of factor B and factor H. J Immunol 177: 1872-1878.

De Vita F (2010) Use of serum VEGF levels to predict outcome of esophageal cancer patients undergoing preoperative chemoradiation. J Clin Oncol 28: 15s suppl; abstract 4140 .

Ferlay J, Soerjomataram I, Ervik M, Dikshit R, Eser S, Mathers C, Rebelo M, Parkin DM, Forman D, Bray F. GLOBOCAN 2012 v1.0, Cancer Incidence and Mortality Worldwide: IARC CancerBase No. 11. Available at http:// globocan.iarc.fr accessed on 15 February 2015.

Fuchs C, Tomasek J, Yong CJ, Dumitru F, Passalacqua R, Goswami C, Safran H, dos Santos LV, Aprile G, Ferry DR, Melichar B, Tehfe M, Topuzov E, Zalcberg JR, Chau I, Campbell W, Sivanandan C, Pikiel J, Koshiji M, Hsu Y, Liepa AM, Gao L, Schwartz JD, Tabernero. REGARD Trial Investigators (2014) REGARD: Ramucirumab monotherapy for previously treated advanced gastric or gastro-oesophageal junction adenocarcinoma 
(REGARD): an international, randomised, multicentre, placebo-controlled, phase 3 trial. Lancet 383: 31-39.

Gan HK, Seruga B, Knox JJ (2009) Sunitinib in solid tumors. Exp Opin Investig Drugs 18: 821-834.

Grivas PD, Daignault S, Tagawa ST, Nanus DM, Stadler WM, Dreicer R, Kohli M, Petrylak DP, Vaughn DJ, Bylow KA, Wong SG, Sottnik JL, Keller ET, Al-Hawary M, Smith DC, Hussain M (2014) Double-blind, randomized, phase 2 trial of maintenance sunitinib versus placebo after response to chemotherapy in patients with advanced urothelial carcinoma. Cancer 120: 692-701.

Lyros O, Mueller A, Heidel F, Schimanski CC, Gockel I, Galle PR, Lang H, Moehler M (2010) Analysis of anti-proliferative and chemosensitizing effects of sunitinib on human esophagogastric cancer cells: synergistic interaction with vandetanib via inhibition of multi-receptor tyrosine kinase pathways. Int J Cancer 127: 1197-1208.

Moehler MH, Thuss-Patience PC, Schmoll HJ, Hegewisch-Becker S, Wilke H, Al-Batran S-E, Weissinger F, Kullmann F, Von Weikersthal LF, Siveke JT, Kanzler S, Schimanski CC, Otte M, Schollenberger L, Koenig J, Galle PR (2013) FOLFIRI plus suntinib versus FOLFIRI alone in advanced chemorefractory esophago-gastric cancer patients: a randomized placebocontrolled multicentric AIO phase II trial. J Clin Oncol 31: suppl; abstract 4086.

Ohtsu A, Shah MA, Van Cutsem E, Rha SY, Sawaki A, Park SR, Lim HY, Yamada Y, Wu J, Langer B, Starnawski M, Kang YK (2011) Bevacizumab in combination with chemotherapy as first-line therapy in advanced gastric cancer: a randomized, double-blind, placebo-controlled phase III study. J Clin Oncol 29: 3968-3976.

Qin S (2014) Phase III study of apatinib in advanced gastric cancer: a randomized, double-blind, placebo-controlled trial. J Clin Oncol 32:5s: suppl; abstract 4003 .

Rini BI, Michaelson MD, Rosenberg JE, Bukowski RM, Sosman JA, Stadler WM, Hutson TE, Margolin K, Harmon CS, DePrimo SE, Kim ST, Chen I, George DJ (2008) Antitumor activity and biomarker analysis of sunitinib in patients with bevacizumab-refractory metastatic renal cell carcinoma. J Clin Oncol 26: 3743-3748.

Schmitt JM, Sommers SR, Fisher W, Ansari R, Robin E, Koneru K, McClean J, Liu Z, Tong Y, Hanna N (2012) Sunitinib plux paclitaxel in patients with advanced esophageal cancer: a phase II study from the Hoosier Oncology Group. J Thorac Oncol 7: 760-763.

Selid PD, Jundt MC, Fortney AC, Beal JR (2014) Intravitreal bevacizumab and aflibercept for the treatment of exudative age-related macular degeneration. Opthalmic Surg Lasers Imaging Retina 45: 275-281.

Shah MA, Ramanathan RK, Ilson DH, Levnor A, D'Adamo D, O'Reilly E, Tse A, Trocola R, Schwartz L, Capanu M, Schwartz GK, Kelsen DP (2006)
Multicenter phase ii study of irinotecan, cisplatin, and bevacizumab in patients with metastatic gastric or gastroesophageal junction adenocarcinoma. J Clin Oncol 24: 5201-5206.

Therasse P, Arbuck SG, Eisenhauer EA, Wanders J, Kaplan RS, Rubinstein L, Verweij J, Van Glabbeke M, van Oosterom AT, Christina MC, Gwyther SG (2000) New guidelines to evaluate the response to treatment in solid tumors. European Organization for Research and Treatment of Cancer, National Cancer Institute of the United States, National Cancer Institute of Canada. J Natl Cancer Inst. 92: 924-930.

Van Cutsem E, de Haas S, Kang YK, Ohtsu A, Tebbutt NC, Ming Xu J, Peng Yong W, Langer B, Delmar P, Scherer SJ, Shah MA (2012) Bevacizumab in combination with chemotherapy as first-line therapy in advanced gastric cancer: a biomarker evaluation from the AVAGAST randomized phase III trial. J Clin Oncol 30: 2119-2127.

Van Groeningen C, Richel D, Giaccone G (2004) Geftinib phase II study in second-line treatment of advanced esophageal cancer. J Clin Oncol 22: suppl abstract 4022 .

Wilke H, Muro K, Van Cutsem E, Oh SC, Bodoky G, Shimada Y, Hironaka S, Sugimoto N, Lipatov O, Kim TY, Cunningham D, Rougier P, Komatsu Y, Ajani J, Emig M, Carlesi R, Ferry D, Chandrawansa K, Schwartz JD, Ohsu A. RAINBOW Study Group (2014) Ramucirumab plus paclitaxel versus placebo plus paclitaxel in patients with previously treated advanced gastric or gastro-esophaeal junction adenocarcinoma (RAINBOW): a doubleblind, randomized phase 3 trial. Lancet Oncol 11: 1224-1235.

Yang X, Liang J, Heverhagen JT, Jia G, Schmalbrock P, Sammet S, Koch R, Knopp MV (2008) Improving the pharmacokinetic parameter measurement in dynamic contrast-enhanced MRI by use of the arterial input function: theory and clinical application. Magn Reson Med 59: 1448-1456.

Zhang H, Wu J, Meng L, Shou CC (2002) Expression of vascular endothelial growth factor and its receptors KDR and Flt-1 in gastric cancer cells. World J Gastroenterol 8: 994-998.

Zhang Q, Yu C, Peng S, Xu H, Wright E, Zhang X, Huo X, Cheng E, Pham TH, Asanuma K, Hatanpaa KJ, Rezai D, Wang DH, Sarode V, Melton S, Genta RM, Spechler SJ, Souza RF (2014) Autocrine VEGF signaling promotes proliferation of neoplastic Barrett's epithelial cells through a PLC-dependent pathway. Gastroenterology 146: 461-472.

This work is published under the standard license to publish agreement. After 12 months the work will become freely available and the license terms will switch to a Creative Commons AttributionNonCommercial-Share Alike 4.0 Unported License.

Supplementary Information accompanies this paper on British Journal of Cancer website (http://www.nature.com/bjc) 